\title{
Alternative lengthening of telomeres phenotype and loss of ATRX expression in sarcomas (Review)
}

\author{
XIAOLEI REN ${ }^{1}$, CHAO TU $^{1}$, ZHENCHU TANG $^{1}$, RUOFEI MA ${ }^{2}$ and ZHIHONG LI $^{1}$ \\ ${ }^{1}$ Department of Orthopaedics, The Second Xiangya Hospital, Central South University, Changsha, Hunan 410011; \\ ${ }^{2}$ Department of Orthopaedics, Beijing Jishuitan Hospital, Beijing 100035, P.R. China
}

Received November 16, 2017; Accepted March 16, 2018

DOI: $10.3892 / \mathrm{ol} .2018 .8318$

\begin{abstract}
Sarcoma is a rare and heterogeneous type of cancer with an early mean onset age and a poor prognosis. However, its genetic basis remains unclear. A series of recent genomic studies in sarcomas have identified the occurrence of mutations in the $\alpha$-thalassemia/mental retardation syndrome X-linked (ATRX) gene. The ATRX protein belongs to the SWI/SNF family of chromatin remodeling proteins, which are frequently associated with $\alpha$-thalassemia syndrome. Cancer cells depend on telomerase or the alternative lengthening of telomeres (ALT) pathway to overcome replicative programmed mortality. Loss of ATRX is associated with ALT in sarcoma. In the present review, recent whole genome and/or whole exome genomic studies are summarized. In addition ATRX immunohistochemistry and ALT fluorescence in situ hybridization in sarcomas of various subtypes and at diverse sites, including osteosarcoma, leiomyosarcoma, liposarcoma, angiosarcoma and chondrosarcoma are evaluated. The present review involves certain studies associated with the molecular mechanisms underlying the loss of ATRX controlling the activation of ALT in sarcomas. Identification of the loss of ATRX and ALT in sarcomas may provide novel methods for the treatment of aggressive sarcomas.
\end{abstract}

\section{Contents}

1. Introduction

2. Loss of ATRX in sarcomas

3. Loss of ATRX is associated with the ALT pathway in sarcoma

Correspondence to: Professor Zhihong Li, Department of Orthopaedics, The Second Xiangya Hospital, Central South University, 139 Middle Renmin Road, Changsha, Hunan 410011, P.R. China

E-mail: lizhihong@csu.edu.cn

Key words: alternative lengthening of telomeres, sarcoma, $\alpha$-thalassemia/mental retardation syndrome $\mathrm{X}$-linked gene
4. Molecular mechanisms of the loss of ATRX controlling the activation of ALT in sarcomas

5. Discussion and conclusion

\section{Introduction}

The $\alpha$-thalassemia/mental retardation syndrome X-linked (ATRX) protein encoded by the ATRX gene (NM_000489: c.6130C>T, p.Leu2044Phe) belongs to the SWI/SNF DNA helicase chromatin remodeling protein family, and contains a plant homeodomain (PHD)-like domain and an ATPase/helicase domain. It was first reported to undergo alterations during cell cycle-dependent phosphorylation, which is closely associated with its nuclear matrix at interphase, condensed chromatin, particularly at the $\mathrm{M}$ phase onset, and chromosomal segregation in mitosis (1).

Mutations in the ATRX gene are associated with a wide and clinically heterogeneous spectrum of X-linked mental retardation (XLMR) syndrome, frequently accompanied by $\alpha$-thalassemia (ATRX) syndrome, a difficultly diagnosed genetic disorder comprising dysmorphic features, microcephaly, severe intellectual disability, cryptorchidism and mild anemia (1). To date, 66 mutations have been reported, primarily located in the PHD-like domain, which is regarded as the major mutation hot-spot (2). These mutations have been demonstrated to induce distinct variation in the pattern of DNA methylation, which may provide a connection between chromatin remodeling and gene expression in dynamic processes. It has been reported that multiple alternatively spliced transcript variants encode diverse isoforms. Several lines of evidence have indicated the effect of ATRX in the regulation of chromatin remodeling, and protein and DNA interactions $(3,4)$. A total of two primary functional domains have been predicted in the light of protein structural motifs (Fig. 1): On the one hand, a PHD-like domain, encoded by exons 7, 8 and 9 (5\# part); and on the other hand, a helicase domain located at the C-terminus, encoded by exons 18, 19, 20 and 21 . These two domains have been reported to serve a role as chromatin remodelers (5). A third domain, predicted as a coiled coil structure, has been demonstrated to interact particularly with EZH2, a protein belonging to the polycomb multigenic family that is involved in histone methylation and deacetylation (6). Mutations in the ATRX gene have been 
identified in recent whole genome and/or whole exome genomic studies in sarcomas, particularly osteosarcoma (7-10).

Telomeres, the short, non-protein coding repeated hexameric TTAGGG sequences at each end of a chromosome, are involved in the control of genome integrity and chromosomal stability, and the development of cancer cells (11). During chromosome replication in somatic cells, telomeres shorten at each duplication, as the synthesis of Okazaki fragments requires RNA primers to attach ahead on the lagging strand so that the gap between the final RNA primer and the end of the chromosome cannot be completed, and 3' overhangs occur (12). Cells may be alive without division until meeting with a barrier called 'crisis', at which a majority of them will undergo programmed cell death. However, pre-malignant cells may cross the crisis barrier by altering the telomere length pathway, leading to cancer initiation by inducing chromosomal instability $(13,14)$. Telomerase activation and the alternative lengthening of telomeres (ALT) pathway have been proposed as the main telomere maintenance mechanisms underlying the eternal proliferation capacity, and blocking genetic disorders induced by telomere dysfunction. The length of telomeres is primarily maintained by telomerase activation or, in $10-20 \%$ of cases, particularly gliomas and sarcomas, by the ALT pathway (15). The activation of ALT reduces tumor relapse in mouse models, although it remains to be elucidated how the molecular mechanisms control the activation of ALT $(16,17)$.

Cancer cells, which resist cellular replicative senescence, become immortal cells by activating telomerase or the ALT pathway $(18,19)$. ALT exists in $5-15 \%$ of all types of human cancer and it is common in specific types of cancer, including osteosarcoma and glioblastoma (20), suggesting that ALT contributes to genomic instability. ATRX is part of a multiprotein complex that regulates telomere maintenance; the loss of the protein ATRX is associated with ALT in cancer, which is frequently mutated in osteosarcoma patients.

Sarcomas are a rare and heterogeneous group of tumors with an earlier mean onset age than other types of epithelial cancer, accounting for $20 \%$ of childhood cancer and $10 \%$ of adolescent and young adult cancer, in addition to various clinical manifestations, differentiations, histopathological types, molecular pathogeneses and prognoses. However, the genetic basis for musculoskeletal sarcoma remains largely unknown (21). Epidemiological studies have identified a firm genetic component of sarcomas and a number of familial cancer syndromes have been reported, which is a characteristic of sarcomas (22). Recently, a published genetic-association study concerning 1,162 patients with sarcoma from four different clinical cohort studies demonstrated that $55 \%$ of these patients had underlying monogenic or polygenic variations. Except TP53, BRCA2, ATM and ataxia telangiectasia and Rad3-related kinase (ATR), a surprisingly excess of functional mutations were identified in ERCC2, which is a transcription factor required for nucleotide excision repair. The greater the accumulated burden of multiple pathogenic variants, the earlier the age at which the cancer was diagnosed. To a certain degree, these data demonstrated that sarcoma has a genetic etiology (23). The musculoskeletal sarcoma National Comprehensive Cancer Network guidelines (www.nccn. org) have recommended genetic testing and consulting for patients with a clinical and/or family history of genetic cancer syndromes associated with a high risk of the development of musculoskeletal sarcoma $(21,24)$.

Cytogenetic variants form one of the earliest and most impactful factors in the classification of musculoskeletal sarcoma. Since the first discovery of the $t(11 ; 22)$ (q24;q12) variant in Ewing sarcoma, a cytogenetic catalog of archives of chromosomal alterations specifying distinct mesenchymal tumor entities has been created (cgap.nci.nih. gov/Chromosomes/Mitelman) (25-27). According to cytogenetic variant evidence, sarcomas may be broadly distributed into two groups: i) Sarcomas with simple karyotypes, frequently involving specific genetic alterations, including specific oncogenic mutations and reciprocal chromosomal translocations; and ii) sarcomas with complex unbalanced karyotypes, usually involving non-specific genetic abnormalities, including chromosomal number alterations, translocations, and large amplifications or deletions that may be illustrated [karyotyping, fluorescence in situ hybridization (FISH)]. The present review summarizes recent whole genome and/or whole exome genomic studies, in addition to ATRX immunohistochemistry and ALT FISH, in sarcomas of various subtypes and in diverse sites, including osteosarcoma, leiomyosarcoma, liposarcoma, angiosarcoma and chondrosarcoma. Additionally, the present review involves a few studies associated with the molecular mechanisms underlying the loss of ATRX controlling the activation of ALT in sarcomas. Testing for the loss of ATRX and ALT in sarcomas may facilitate the identification of novel targets for the treatment of aggressive sarcomas.

\section{Loss of ATRX in sarcomas}

Mutations in the ATRX gene have been discovered in a series of sarcomas, including osteosarcoma, leiomyosarcoma and chondrosarcoma. The first report of diagnosed osteosarcoma in two brothers with ATRX syndrome suggested a potential increased risk of cancer in patients with this disorder (28). A 22-year-old Caucasian man was reported in 2017 to exhibit a previously unidentified mutation in ATRX associated with osteosarcoma (29). All these data suggested that patients with ATRX syndrome may be at a potential increased risk of developing osteosarcoma, although the molecular mechanism of ATRX loss-of-function mutations in osteosarcoma remains unclear at present $(30,31)$.

Long-term studies by Liau et al have provided evidence of the frequency of ATRX expression loss in 519 sarcomas samples (30). Those studies identified 85 tumors in those samples $(85 / 519,16 \%)$ arising from ATRX loss, consisting of 83 sarcomas with complex cytogenetics and two sarcomas with fusion genes; the details of the sarcoma types are presented in Table I. The loss of ATRX expression with complex cytogenetics was significantly more frequent in sarcoma compared with fusion-associated sarcoma (30). The previous studies of Liau et al reported additional details. For example, the loss of ATRX in leiomyosarcoma was correlated with cell modalism, poor differentiation, necrosis, estrogen receptor expression, lower patient age and smaller tumor size. There was no significant association with tumor site, compared among uterine and non-uterine, non-retroperitoneal or non-intra-abdominal sites (32). 


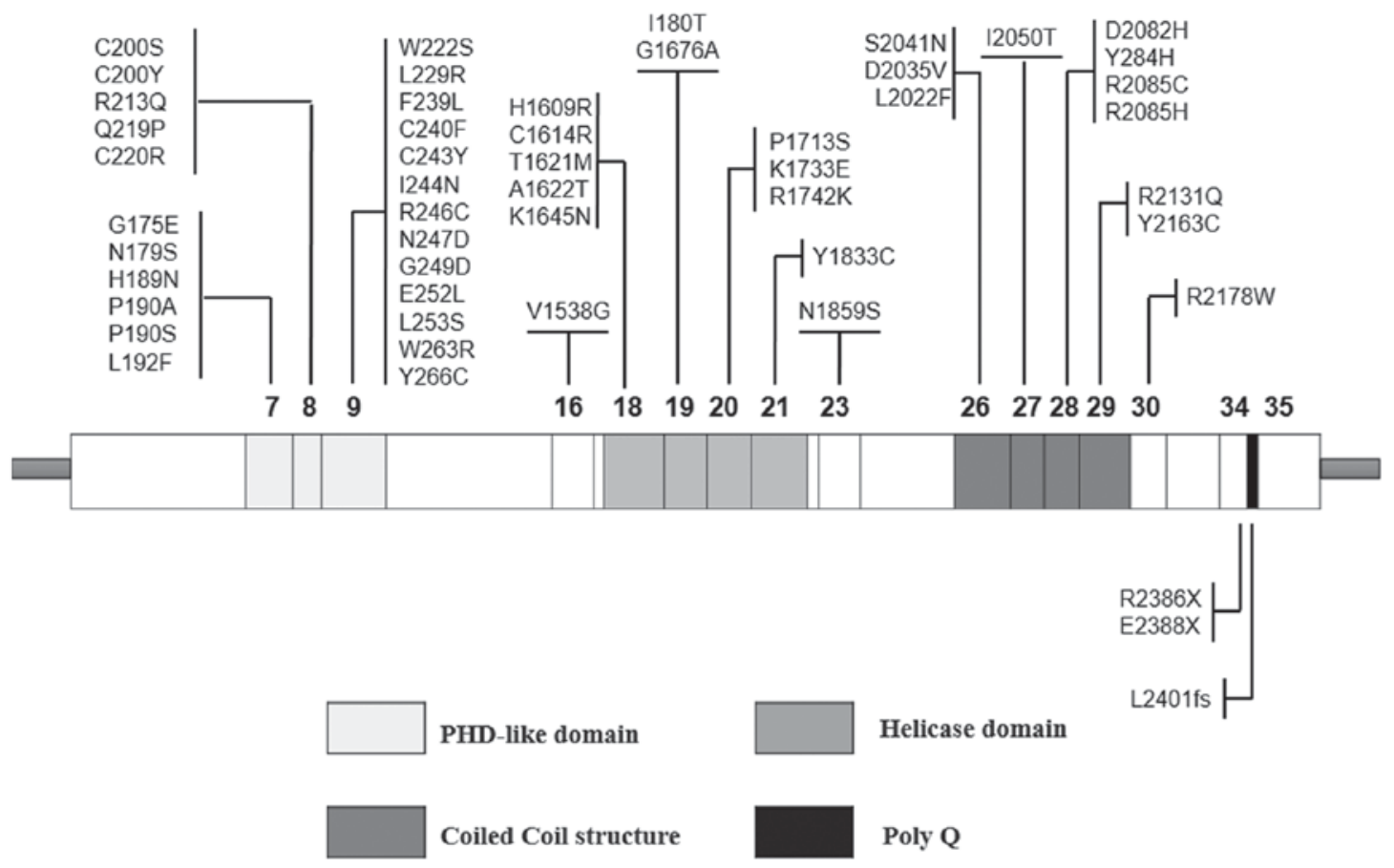

Figure 1. Diagram of the structure of $\alpha$-thalassemia/mental retardation syndrome X-linked cDNA. The boxes represent the 35 exons. The position of the PHD-like domain, the helicase domain and the coiled-coil motif are illustrated with different colors. Positions and types of mutation obtained from the literature are additionally presented. PHD, plant homeodomain.

Furthermore, an osteosarcoma discovery cohort identified the somatic mutation landscapes of 34 primary and metastatic pediatric osteosarcomas via whole-genome sequencing, reported Chen et al (33). A total of five osteosarcomas exhibited ATRX point mutations, and five exhibited structural variations or focal deletions impacting the ATRX gene coding regions. In addition, upon analysis with immunohistochemistry (IHC), $69 \%(13 / 19)$ of the tumors were ATRX-positive.

A series of studies have used a next generation sequencing (NGS) panel to examine common cancer-associated genetic alterations. Lee et al (34) reported that 25 leiomyosarcomas occurring in multiple sites were associated with the frequent gene alterations in the following proportions: TP53 (36\%), ATM and ATRX (16\%), and EGFR and RB1 (12\%). Furthermore, Mäkinen et al (35) reported that 43 genes exhibited mutations in 19 uterine leiomyosarcoma (ULMS) tumors, including the following frequently mutant genes: TP53 (6/19, $33 \%), \operatorname{ATRX}(5 / 19,26 \%)$, and mediator complex subunit 12 (MED12; 4/19, 21\%), demonstrated by whole-exome sequencing. However, all the ATRX alterations were either frameshift or nonsense mutations, opposite to TP53 and MED12 which are all the identified alterations. In addition, ATRX protein expression levels were analyzed by IHC in a total of 44 ULMS tumors, indicating markedly reduced ATRX expression in 23 tumors (23/44, 52\%). Yang et al (36) analyzed the genetic alterations in 44 cancer-associated genes via NGS in 54 leiomyosarcomas. The most frequently mutated genes were identified, including TP53 mutations in 19 of the leiomyosarcomas $(19 / 54,35 \%)$ and ATRX mutations in 9 of the tumors $(9 / 54,17 \%)$. Notably, the ATRX mutations were associated with low-differentiation or undifferentiated leiomyosarcomas $(\mathrm{P}=0.028)$, and the existence of tumor necrosis $(\mathrm{P}=0.015)$. In addition, leiomyosarcoma patients with ATRX mutations exhibited a poorer prognosis compared with ATRX-wild-type patients, as demonstrated by Kaplan-Meier survival analysis.

Hartmann et al (37) reported that isocitrate dehydrogenase (IDH)-1 or -2 mutations have been detected in gliomas (60-80\%) and cholangiocarcinomas (7-28\%). Notably, the loss of ATRX occurred in the IDH-mutant gliomas. However, without relevance in cholangiocarcinomas was evaluated by IHC 2 of $36(5.6 \%)$ and usual chondrosarcomas showed complete negative of ATRX (38).

\section{Loss of ATRX is associated with the ALT pathway in sarcoma}

Previous studies have suggested that the ALT pathway exists in the majority of cancer types. Previous studies analyzed the frequency of ALT correlating with the loss of ATRX in soft tissue sarcomas. Liau et al characterized the significant association between the ALT pathway and ATRX status in 519 sarcoma samples $(\mathrm{P}<0.001)(30)$. It was reported that the association was relatively similar in undifferentiated pleomorphic sarcoma, leiomyosarcoma and pleomorphic liposarcoma (Table I). Notably, over 50\% tumors are identified to be ALT-positive in which ATRX deficient were observed about $50 \%$. By contrast, only a few malignant peripheral nerve sheath tumors and embryonal rhabdomyosarcomas were ALT-positive or exhibited a loss of ATRX (or both), which indicated that ALT status and the loss of ATRX were likely to be associated with katagenesis and dedifferentiation variation (30). Additionally, these previous studies included certain essential information. For example, deletion of ATRX was observed in $33 \%$ of the leiomyosarcomas, and all of them were ALT-positive except 
Table I. The proportions of ATRX loss and ALT status in sarcomas with complex cytogenetics and fusion genes.

\begin{tabular}{lcccc}
\hline & & & \multicolumn{2}{c}{ ALT $(+)$} \\
\cline { 4 - 5 } Sarcomas with complex cytogenetics & $\mathrm{N}$ & ATRX loss $(\%)$ & $\mathrm{N}(\%)$ & ATRX loss (\%) \\
\hline Leiomyosarcoma & 92 & $30(33)$ & $51 / 86(59)$ & $28 / 51(55)$ \\
Angiosarcoma & 88 & $16(18)$ & $17 / 70(24)$ & $15 / 17(88)$ \\
Dedifferentiated liposarcoma & 52 & $13(25)$ & $14 / 46(30)$ & $13 / 14(93)$ \\
Undifferentiated pleomorphic sarcoma & 35 & $12(34)$ & $22 / 34(65)$ & $12 / 22(55)$ \\
Myxofibrosarcoma & 27 & $1(4)$ & $19 / 25(76)$ & $1 / 19(5)$ \\
Radiation-associated sarcoma & 20 & $0(0)$ & $3 / 15(20)$ & $0 / 3(0)$ \\
Osteosarcoma & 18 & $4(22)$ & $\mathrm{N} / \mathrm{A})$ & $\mathrm{N} / \mathrm{A}$ \\
Malignant peripheral nerve sheath tumor & 17 & $1(6)$ & $3 / 14(21)$ & $1 / 3(33)$ \\
Embryonal rhabdomyosarcoma & 9 & $1(11)$ & $1 / 8(13)$ & $1 / 1(100)$ \\
Pleomorphic liposarcoma & 11 & $5(45)$ & $8 / 10(80)$ & $5 / 8(63)$ \\
Sarcomas with fusion genes & & $1(9)$ & $1 / 7(14)$ & $1 / 1(100)$ \\
Epithelioid hemangioendothelioma & 11 & $1(4)$ & $1 / 16(6)$ & $1 / 1(100)$ \\
Gastrointestinal stromal tumor & 23 & & & \\
\hline
\end{tabular}

ATRX, $\alpha$-thalassaemia/intellectual disability syndrome X-linked; ALT, alternative lengthening of telomeres; N/A, not applicable.

for two ALT-negative tumors out of all the ATRX-deletion tumors. The ALT phenotype and ATRX deletion are both associated with cell morphology, tumor necrosis and differentiation. In addition, ALT-positivity and younger patient age were independent poor-prognostic risk factors in the multivariate analysis (32). Furthermore, previous studies have reported that ALT was detected in 20-30\% of liposarcomas (20,39). Notably, Lee et al (34) provided evidence of a perfect correlation between the loss of either ATRX or death domain-associated protein and the ALT status in 46 dedifferentiated liposarcoma samples (DDLS) via telomere-specific FISH and IHC, and the ALT status significantly indicated poor clinical outcomes, including poor overall survival and short progression-free survival $(40,41)$. Notably, all the well-differentiated (WD) liposarcomas were ALT-negative $(40,42)$.

Yang et al (36) reported that all ATRX-mutated leiomyosarcoma samples exhibited the ALT phenotype $(\mathrm{P}=0.008)$. Although the ATRX mutation was not correlated with the loss of ATRX expression, all the tumors with nonsense and frame shift mutations were ATRX-negative, according to IHC (36). Likewise, the loss of ATRX expression was confirmed in all uterine leiomyosarcoma samples. The loss of ATRX expression has been associated with ALT, as analyzed by telomere-specific FISH (35).

Similarly, myxofibrosarcoma was frequently positive for the ALT pathway (43). Recently, a KRAS-mutated KIT/PDGFRA-wild-type gastrointestinal stromal tumor was revealed to exhibit ATRX mutations (44). This tumor also exhibited anaplastic histological features and behaved aggressively, which indicates that the loss of ATRX expression and/or the acquisition of the ALT pathway may be associated with dedifferentiation and/or transdifferentiation of gastrointestinal stromal tumors, indicating a poor prognosis.
Similarly, Chen et al (33) demonstrated that 85\% (12/14) of pediatric osteosarcomas used the ALT mechanism to maintain their telomeres detected by the WGS data, telomeres quantitative polymerase chain reaction and telomere-specific FISH. Furthermore, 58\% of 12 ALT-positive osteosarcomas exhibited ATRX mutations.

By contrast, a previous study by Heaphy et al (20) reported that two out of six epithelioid sarcomas were ALT-positive, and none of these tumors were revealed to be ALT and ATRX deficient, implying that marked genetic alterations may have promoted tumor formation instead of the ALT mechanism in this series of tumors. Consequently, these were more similar to the fusion-associated sarcomas and gastrointestinal stromal tumors in a study undertaken by Liau et al (30).

\section{Molecular mechanisms of the loss of ATRX controlling the activation of ALT in sarcomas}

As mentioned earlier, the loss of ATRX expression is associated with ALT, but the molecular mechanisms controlling the activation of ALT remain unclear. Previous studies into the molecular mechanisms involved in sarcomas have been rare. Napier et al (45) primarily reported the direct and functional evidence that ATRX loss accelerates ALT. In the aforementioned study, the knockdown of ATRX in pre-crisis simian virus 40-transformed fibroblasts led to activated ALT or a significant decrease in the time prior to ALT activation. Furthermore, transiently upregulated ATRX expression was able to suppress ALT activity in ALT-positive cells.

Additionally, Flynn et al (46) reported that the loss of ATRX leads to the formation of a recombinogenic nucleoprotein complex consisting of ATR, replication protein A (RPA), and telomeric non-coding RNA. The inhibition of ATR, an essential recombination regulator recruited by RPA, disrupts 


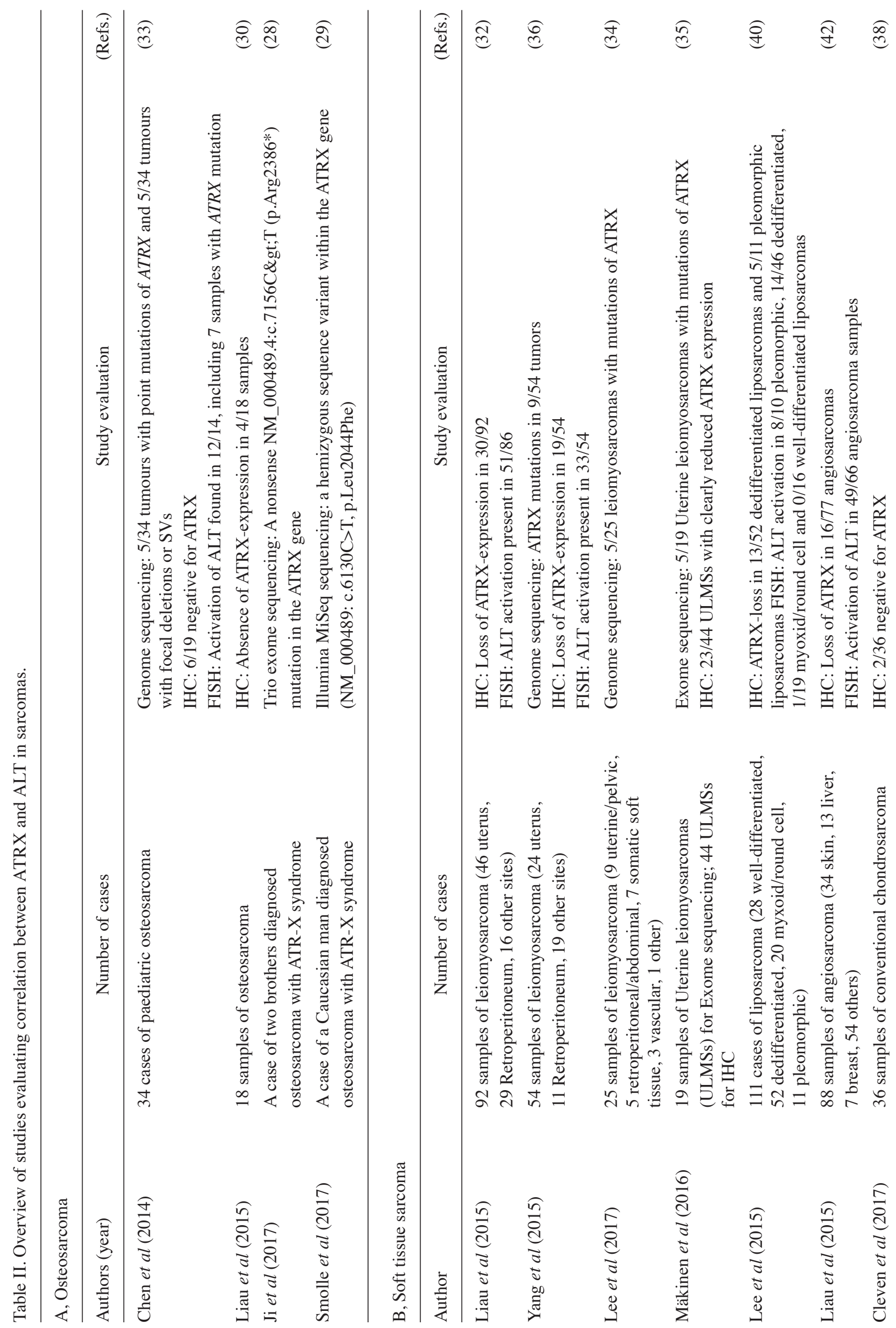


ALT, and causes chromosome disruption and apoptosis in ALT-positive cells. Notably, cell death induced by ATR inhibitors is highly selective for ALT-positive cancer cells, proposing that ATR inhibitors may be used to treat ALT-positive cancer. At present, there have been two highly selective and effective ATR inhibitors, AZD6738 and VX-970, which have reached phase I clinical trials. These drugs are being used either as a monotherapy or combined with a series of genotoxic chemotherapies for the treatment of ALT-positive solid tumors and refractory cancer (47).

Furthermore, a series of studies have revealed the possible mechanism underlying the manner in which ATRX loss leads to sarcoma formation. McFarlane and Preston (48) proved that the presence of ATRX inhibited the expression of human cytomegalovirus immediate early genes instead of herpes simplex virus type 1 in the osteosarcoma U2OS cell line, and the specific virus phosphoprotein PP71 was able to withstand the inhibition due to the disassembly of ATRX with nuclear domain 10 in human fetal foreskin fibroblast 2 cells. Kovatcheva et al (49) revealed that the turnover of mouse double minute 2 homolog (MDM2) and the expression of ATRX were crucial in WD and DDLS cell lines. Particularly, the effect of the ATRX expression is significant in cyclin-dependent kinase 4 (CDK4) inhibitors responder inducing silence or consenescence. ATRX deficiency prevents the loss of MDM2, which may be blocked by ATRX $\mathrm{C}$-terminal modifications. This discovery may directly guide the effective enhancement of CDK4 inhibitors in clinical cancer therapy.

\section{Discussion and conclusion}

The present review presented screening data for ALT status and ATRX alteration from a variety of sarcomas (Table II). It was surmised that recent whole genome and/or whole exome genomic studies, in addition to ATRX immunohistochemistry and ALT FISH, have been performed for various sarcomas in diverse sites, including osteosarcoma, leiomyosarcoma, liposarcoma, angiosarcoma and chondrosarcoma. Considering the results of these studies, it has been demonstrated that ALT serves an essential role in telomere maintenance in sarcomas, and is continually affected by ATRX expression loss. However, the correlation between ALT and ATRX expression loss differs among the sarcoma types and the differences are distinct. For certain tumors, particularly the myxofibrosarcomas, it appears that the mechanisms may be ascribed to ALT instead of the loss of ATRX expression in the majority of cases.

Recently, the cell death induced by ATR inhibitors, which were developed as feasible novel drugs for ATRX-deficient tumors, have demonstrated high selectivity for ALT-positive cancer cells, indicating that such types of inhibitors may be effective for the treatment of cancer with an ALT-dependent mechanism $(46,48)$. ATR was reported to be crucial for homologous recombination and ALT, and inhibition of this kinase caused apoptosis in ALT-positive cancer cells. This demonstrates the significance and development of clinical genome analysis to assist diagnosis and facilitate individualized precision targeted therapies for sarcomas with complex pathologies. Identification of an ALT phenotype and the loss 
of ATRX are not rarely seen in sarcomas, suggesting that ATR inhibitors may provide a novel option to treat these invasive neoplasms $(46,48)$.

\section{Acknowledgements}

Not applicable.

\section{Funding}

This study was supported by the National Natural Science Foundation of China (grant. no. 81372180) and the Hunan Provincial Science and Technology Association program (grant no. 2017TJ-Q19) and the Hunan Provincial Science and Technology Association key-point program (grant no. 2017DK2013).

\section{Availability of data and materials}

The datasets used and/or analyzed during the current study are available from the corresponding author on reasonable request.

\section{Authors' contributions}

$\mathrm{XR}, \mathrm{RM}$ and ZT collected the associated articles about the ATRX gene. XR and CT analysed and summarized the data. $\mathrm{XR}$ wrote the paper and edited the manuscript and agreed to be accountable for all aspects of the work ensuring that questions related to the accuracy or integrity of any part of the work are appropriately investigated and resolved. CT analyzed and summarized the data. XR was a major contributor in writing the manuscript. ZL revised and checked the manuscript and gave the final approval of the version to be published. All authors read and approved the final manuscript.

\section{Ethics approval and consent to participate}

Not applicable.

\section{Consent for publication}

Not applicable.

\section{Competing interests}

The authors declare that they have no competing interests.

\section{References}

1. Gibbons RJ, Picketts DJ, Villard L and Higgs DR: Mutations in a putative global transcriptional regulator cause X-linked mental retardation with alpha-thalassemia (ATR-X syndrome). Cell 80 : 837-845, 1995.

2. Villard L and Fontes M: Alpha-thalassemia/mental retardation syndrome, X-Linked (ATR-X, MIM \#301040, ATR-X/XNP/XH2 gene MIM \#300032). Eur J Hum Genet 10: 223-225, 2002.

3. Qadeer ZA, Harcharik S, Valle-Garcia D, Chen C, Birge MB, Vardabasso C, Duarte LF and Bernstein E: Decreased expression of the chromatin remodeler ATRX associates with melanoma progression. J Invest Dermatol 134: 1768-1772, 2014.

4. Cai J, Chen J, Zhang W, Yang P, Zhang C, Li M, Yao K, Wang H, Li Q, Jiang C and Jiang T: Loss of ATRX, associated with DNA methylation pattern of chromosome end, impacted biological behaviors of astrocytic tumors. Oncotarget 6: 18105-18115, 2015.
5. Gibbons RJ and Higgs DR: Molecular-clinical spectrum of the ATR-X syndrome. Am J Med Genet 97: 204-212, 2000.

6. Cardoso C, Timsit S, Villard L, Khrestchatisky M, Fontès M and Colleaux L: Specific interaction between the XNP/ATR-X gene product and the SET domain of the human EZH2 protein. Hum Mol Genet 7: 679-684, 1998.

7. Perry JA, Kiezun A, Tonzi P, Van Allen EM, Carter SL, Baca SC, Cowley GS, Bhatt AS, Rheinbay E, Pedamallu CS, et al: Complementary genomic approaches highlight the PI3K/mTOR pathway as a common vulnerability in osteosarcoma. Proc Natl Acad Sci USA 111: E5564-E5573, 2014.

8. Reimann E, Kõks S, Ho XD, Maasalu K and Märtson A: Whole exome sequencing of a single osteosarcoma case-integrative analysis with whole transcriptome RNA-seq data. Hum Genomics 8: 20, 2014.

9. Bousquet M, Noirot C, Accadbled F, Sales de Gauzy J, Castex MP, Brousset $\mathrm{P}$ and Gomez-Brouchet A: Whole-exome sequencing in osteosarcoma reveals important heterogeneity of genetic alterations. Ann Oncol 27: 738-744, 2016.

10. Joseph CG, Hwang H, Jiao Y, Wood LD, Kinde I, Wu J, Mandahl N, Luo J, Hruban RH, Diaz LA Jr, et al: Exomic analysis of myxoid liposarcomas, synovial sarcomas, and osteosarcomas. Genes Chromosomes Cancer 53: 15-24, 2014.

11. Li D, Yuan Q and Wang W: The role of telomeres in musculoskeletal diseases. J Int Med Res 40: 1242-1250, 2012.

12. Ozturk MB, Li Y and Tergaonkar V: Current insights to regulation and role of telomerase in human diseases. Antioxidants (Basel) 6: pii: E17, 2017.

13. Henderson ER and Blackburn EH: An overhanging 3' terminus is a conserved feature of telomeres. Mol Cell Biol 9: 345-348, 1989.

14. Hayflick L and Moorhead PS: The serial cultivation of human diploid cell strains. Exp Cell Res 25: 585-621, 1961.

15. Shay JW: Role of telomeres and telomerase in aging and cancer. Cancer Discov 6: 584-593, 2016.

16. Günes C and Rudolph KL: The role of telomeres in stem cells and cancer. Cell 152: 390-393, 2013.

17. Harley CB and Villeponteau B: Telomeres and telomerase in aging and cancer. Curr Opin Genet Dev 5: 249-255, 1995.

18. Artandi SE and DePinho RA: Telomeres and telomerase in cancer. Carcinogenesis 31: 9-18, 2010.

19. Cesare AJ and Reddel RR: Alternative lengthening of telomeres: Models, mechanisms and implications. Nat Rev Genet 11: 319-330, 2010

20. Heaphy CM, Subhawong AP, Hong SM, Goggins MG, Montgomery EA, Gabrielson E, Netto GJ, Epstein JI, Lotan TL, Westra WH, et al: Prevalence of the alternative lengthening of telomeres telomere maintenance mechanism in human cancer subtypes. Am J Pathol 179: 1608-1615, 2011.

21. Sidaway P: Sarcoma: Genetic determinants of sarcoma risk revealed. Nat Rev Clin Oncol 13: 590, 2016.

22. Thomas DM and Ballinger ML: Diagnosis and management of hereditary sarcoma. Recent Results Cancer Res 205: 169-189, 2016.

23. Ballinger ML, Goode DL, Ray-Coquard I, James PA, Mitchell G, Niedermayr E, Puri A, Schiffman JD, Dite GS, Cipponi A, et al: Monogenic and polygenic determinants of sarcoma risk: An international genetic study. Lancet Oncol 17: 1261-1271, 2016.

24. Antonescu CR: The role of genetic testing in soft tissue sarcoma. Histopathology 48: 13-21, 2006.

25. Bridge JA: The role of cytogenetics and molecular diagnostics in the diagnosis of soft-tissue tumors. Mod Pathol 27 (Suppl 1): S80-S97, 2014.

26. Rosenberg AE: WHO classification of soft tissue and bone, fourth edition: Summary and commentary. Curr Opin Oncol 25: 571-573, 2013.

27. Zambo I and Veselý K: WHO classification of tumours of soft tissue and bone 2013: The main changes compared to the 3rd edition. Cesk Patol 50: 64-70, 2014.

28. Ji J, Quindipan C, Parham D, Shen L, Ruble D, Bootwalla M, Maglinte DT, Gai X, Saitta SC, Biegel JA and Mascarenhas L: Inherited germline ATRX mutation in two brothers with ATR-X syndrome and osteosarcoma. Am J Med Genet A 173: 1390-1395, 2017.

29. Smolle MA, Heitzer E, Geigl JB, Al Kaissi A, Liegl-Atzwanger B, Seidel MG, Holzer LA and Leithner A: A novel mutation in ATRX associated with intellectual disability, syndromic features, and osteosarcoma. Pediatr Blood Cancer 64: e26522, 2017.

30. Liau JY, Lee JC, Tsai JH, Yang CY, Liu TL, Ke ZL, Hsu HH and Jeng YM: Comprehensive screening of alternative lengthening of telomeres phenotype and loss of ATRX expression in sarcomas. Mod Pathol 28: 1545-1554, 2015. 
31. Kovac M, Blattmann C, Ribi S, Smida J, Mueller NS, Engert F Castro-Giner F, Weischenfeldt J, Kovacova M, Krieg A, et al: Exome sequencing of osteosarcoma reveals mutation signatures reminiscent of BRCA deficiency. Nat Commun 6: 8940, 2015.

32. Liau JY, Tsai JH, Jeng YM, Lee JC, Hsu HH and Yang CY: Leiomyosarcoma with alternative lengthening of telomeres is associated with aggressive histologic features, loss of ATRX expression, and poor clinical outcome. Am J Surg Pathol 39: 236-244, 2015

33. Chen X, Bahrami A, Pappo A, Easton J, Dalton J, Hedlund E, Ellison D, Shurtleff S, Wu G, Wei L, et al: Recurrent somatic structural variations contribute to tumorigenesis in pediatric osteosarcoma. Cell Rep 7: 104-112, 2014.

34. Lee PJ, Yoo NS, Hagemann IS, Pfeifer JD, Cottrell CE, Abel HJ and Duncavage EJ: Spectrum of mutations in leiomyosarcomas identified by clinical targeted next-generation sequencing. Exp Mol Pathol 102: 156-161, 2017

35. Mäkinen N, Aavikko M, Heikkinen T, Taipale M, Taipale J, Koivisto-Korander R, Bützow R and Vahteristo P: Exome sequencing of uterine leiomyosarcomas identifies frequent mutations in TP53, ATRX, and MED12. PLoS Genet 12: e1005850, 2016.

36. Yang CY, Liau JY, Huang WJ, Chang YT, Chang MC, Lee JC, Tsai JH, Su YN, Hung CC and Jeng YM: Targeted next-generation sequencing of cancer genes identified frequent TP53 and ATRX mutations in leiomyosarcoma. Am J Transl Res 7: 2072-2081, 2015.

37. Hartmann C, Meyer J, Balss J, Capper D, Mueller W, Christians A, Felsberg J, Wolter M, Mawrin C, Wick W, et al: Type and frequency of IDH1 and IDH2 mutations are related to astrocytic and oligodendroglial differentiation and age: A study of 1,010 diffuse gliomas. Acta Neuropathol 118: 469-474, 2009.

38. Cleven AHG, Suijker J, Agrogiannis G, Briaire-de Bruijn IH Frizzell N, Hoekstra AS, Wijers-Koster PM, Cleton-Jansen AM and Bovée JVMG: IDH1 or -2 mutations do not predict outcome and do not cause loss of 5-hydroxymethylcytosine or altered histone modifications in central chondrosarcomas. Clin Sarcoma Res 7: 8, 2017.

39. Johnson JE, Varkonyi RJ, Schwalm J, Cragle R, Klein-Szanto A, Patchefsky A, Cukierman E, von Mehren M and Broccoli D: Multiple mechanisms of telomere maintenance exist in liposarcomas. Clin Cancer Res 11: 5347-5355, 2005.
40. Lee JC, Jeng YM, Liau JY, Tsai JH, Hsu HH and Yang CY: Alternative lengthening of telomeres and loss of ATRX are frequent events in pleomorphic and dedifferentiated liposarcomas. Mod Pathol 28: 1064-1073, 2015.

41. Venturini L, Motta R, Gronchi A, Daidone M and Zaffaroni N: Prognostic relevance of ALT-associated markers in liposarcoma: A comparative analysis. BMC Cancer 10: 254, 2010.

42. Liau JY, Tsai JH, Yang CY, Lee JC, Liang CW, Hsu HH and Jeng YM: Alternative lengthening of telomeres phenotype in malignant vascular tumors is highly associated with loss of ATRX expression and is frequently observed in hepatic angiosarcomas. Hum Pathol 46: 1360-1366, 2015.

43. Willems SM, Debiec-Rychter M, Szuhai K, Hogendoorn PC and Sciot R: Local recurrence of myxofibrosarcoma is associated with increase in tumour grade and cytogenetic aberrations, suggesting a multistep tumour progression model. Mod Pathol 19: 407-416, 2006.

44. Hechtman JF, Zehir A, Mitchell T, Borsu L, Singer S, Tap W, Oultache A, Ladanyi M and Nafa K: Novel oncogene and tumor suppressor mutations in KIT and PDGFRA wild type gastrointestinal stromal tumors revealed by next generation sequencing. Genes Chromosomes Cancer 54: 177-184, 2015.

45. Napier CE, Huschtscha LI, Harvey A, Bower K, Noble JR, Hendrickson EA and Reddel RR: ATRX represses alternative lengthening of telomeres. Oncotarget 6: 16543-16558, 2015.

46. Flynn RL, Cox KE, Jeitany M, Wakimoto H, Bryll AR, Ganem NJ, Bersani F, Pineda JR, Suvà ML, Benes $\mathrm{CH}$, et al: Alternative lengthening of telomeres renders cancer cells hypersensitive to ATR inhibitors. Science 347: 273-277, 2015.

47. Deeg KI, Chung I, Bauer C and Rippe K: Cancer cells with alternative lengthening of telomeres do not display a general hypersensitivity to ATR inhibition. Front Oncol 6: 186, 2016.

48. McFarlane S and Preston CM: Human cytomegalovirus immediate early gene expression in the osteosarcoma line U2OS is repressed by the cell protein ATRX. Virus Res 157: 47-53, 2011.

49. Kovatcheva M, Liu DD, Dickson MA, Klein ME, O'Connor R, Wilder FO, Socci ND, Tap WD, Schwartz GK, Singer S, et al: MDM2 turnover and expression of ATRX determine the choice between quiescence and senescence in response to CDK4 inhibition. Oncotarget 6: 8226-8243, 2015. 